1 Universidade de São Paulo (USP), Faculdade de Saúde Pública - São Paulo (SP), Brasil.

jose.alexandre.bw@gmail.com

2 Universidade de São Paulo (USP), Faculdade de Saúde Pública - São Paulo (SP), Brasil.

aquilasn@uol.com.br

\section{O Orçamento por Desempenho como ferramenta para gestão e avaliação da política de saúde no município de São Bernardo do Campo, no período 2006 a 2012}

The Budget for Performance as a tool for management and evaluation of health policy in the municipality of São Bernardo do Campo, from 2006 to 2012

José Alexandre Buso Weiller', Áquilas Nogueira Mendes²

RESUMO Este artigo tem como objetivo avaliar o desempenho do gasto em saúde do município de São Bernardo do Campo, no período de 2006 a 2012, a partir de uma metodologia de desempenho do orçamento na saúde que busque relacionar o planejamento com os resultados da política de saúde alcançados. Trabalha-se com os instrumentos orçamentários Planos Plurianuais (PPAs), Leis de Diretrizes Orçamentárias (LDOs), Leis Orçamentárias Anuais (LOAs) e, ainda, relatórios e planilhas provenientes dos processos de pactuação em saúde. Assim, utiliza-se uma metodologia baseada no instrumento Orçamento por Desempenho, que permite avaliar a relação entre as metas alcançadas no município de São Bernardo do Campo, os recursos envolvidos e os resultados das políticas de saúde sob a ótica do orçamento.

PALAVRAS-CHAVE Orçamentos. Avaliação de desempenho. Avaliação em saúde. Sistema Único de Saúde.

ABSTRACT This article aims to evaluate the performance of health expenditure of the municipality of São Bernardo do Campo, in the period 2006-2012, from a methodology of the budget's performance in health which seeks to relate the planning with the results of health policy achieved. It is used the budgetary instruments Multiannual Plans (PPAs), Budget Guidelines Laws (LDOs), Annual Budgetary Laws (LOAs) and, also, reports and spreadsheets from the pact processes in health. Thus, the article uses a methodology based on the instrument 'Budget for Performance', which allows evaluate the relationship between the goals achieved in the municipality of São Bernardo do Campo, the resources involved and the results of the health policies from the perspective of the budget.

KEYWORDS Budgets. Performance evaluation. Health evaluation. Unified Health System. 


\section{Introdução}

A política de saúde, com vistas a um processo de mudança e melhora das condições de vida da população, deve vir sempre acompanhada de um contexto que apresente um modo de gestão dessa ação pública e seus respectivos monitoramento e avaliação. Pensar o agir dos entes públicos é estabelecer, a partir das políticas, um constante processo de gestão que envolva o planejamento, o orçamento e seus respectivos controles e avaliações.

Segundo Paim e Teixeira (2006), a política de saúde abrange questões relativas ao poder em saúde, referenciando-se ao estabelecimento de diretrizes, planos e programas, e, ainda, expressa dimensões do poder. Isso pode indicar que a política de saúde abrange as relações de poder na conformação da agenda, na formulação, na condução, na implementação e na avaliação de políticas.

Assim, essa forma de pensar a política de saúde envolve estudos sobre o papel do Estado, a relação Estado-sociedade, as reações às condições de saúde da população e aos seus determinantes. Ampliando-se a análise, torna-se fundamental a elaboração de estudos da política de saúde que busquem relacionar as políticas econômicas e sociais e o controle social, no campo da economia da saúde.

Nessa perspectiva, é preciso ressaltar que os princípios e as diretrizes do Sistema Único de Saúde (SUS), previstos na Constituição Federal de 1988, asseguram uma base para a execução da política de saúde. Da mesma forma, $o$ artigo $\mathrm{n}^{\circ} 165$ dessa Constituição estabelece os instrumentos do processo orçamentário público, o Plano Plurianual (PPA), a Lei de Diretrizes Orçamentárias (LDO) e a Lei Orçamentária Anual (LOA), que compreendem as metas e prioridades a serem executadas da administração pública, em geral, e da saúde, em particular.

Quando considerada a avaliação econômica da saúde, Bonacim e Araujo (2011) indicam que esse processo assume um papel de destaque em todo o mundo, exigindo dos gestores governamentais novos desafios na busca contínua da eficiência e da eficácia das suas atividades. As informações de orçamento e custos associadas aos indicadores de produção e qualidade, embora insuficientes para a visão de conjunto institucional, são importantes no planejamento, na tomada de decisão dos gestores e na avaliação do desempenho da política de saúde.

Segundo Campos (1994, P. 65), "a gestão de um sistema de saúde considera importante o planejamento como instrumento gerencial para ordenar a produção de serviços de saúde". Assim, o planejamento deve contribuir para alcançar melhores alocação e distribuição de recursos, utilizando como ferramentas os diagnósticos epidemiológicos e das necessidades de saúde. Por essa perspectiva, é possível afirmar que a política de saúde é eficaz quando a ação planejada for realizada.

Para Mendes e Santos (2001), o Estado necessita de ferramentas gerenciais ágeis e competentes para que as políticas públicas, por ele implementadas, sejam eficientes e eficazes. Uma política é eficiente quando gasta menos tempo e recursos (financeiros, materiais e humanos) para a sua realização. Para tanto, espera-se que a atuação do Estado se paute pelo princípio da racionalidade, isto é, da otimização de recursos, que só é possível a partir de um planejamento que envolva a solução de problemas e o aproveitamento das potencialidades existentes.

Assim, um novo modelo de gestão orçamentário-financeira que contemple uma lógica de relação entre planejamento da política de saúde e orçamento deve ser pensado à luz de um ciclo do conceito de gestão mais ampliado. Essa ideia é marcada pelas contribuições de Mendes e Santos (2001). Para esses autores, esse novo modelo, então, deve seguir uma lógica que compreenda a relação entre as funções de planejamento, orçamento, execução orçamentária, acompanhamento, controle público (exercido pela sociedade como 
um todo) e a avaliação. Dessa forma, a gestão orçamentário-financeira deve ser encarada como um instrumento de integração dessas funções aos objetivos, produtos e resultados das políticas públicas.

Nesse sentido, uma interessante ferramenta que relaciona o orçamento e os reais impactos da política planejada refere-se ao Performance Budget (PB), utilizado neste trabalho a partir da produção de Robinson (2008). O PB tem a função básica de relacionar, a partir de diferentes eixos de avaliação com indicadores específicos, os recursos despendidos em ações públicas e seus respectivos resultados, visando aos reais impactos nas condições de vida da sociedade.

Um movimento importante realizado pela Organização para a Cooperação e Desenvolvimento Econômico (OECD) foi a publicação de um livro, em 2009, que relatou e discutiu os processos históricos de desenvolvimento da gestão e do Orçamento por Desempenho nos países que compõem essa organização. Tal livro, 'Performance Budgeting in OECD countries' (2007), indica em seu prefácio:

Os governos estão sob pressão para avaliar o seu próprio desempenho. A informação sobre o desempenho do setor público pode satisfazer a necessidade pública de saber, e pode ser usada para mostrar que os governos fornecem uma boa relação custo-benefício através de suas ações. Talvez o mais importante, a informação sobre o desempenho tenha o potencial de ajudar os governantes a tomarem decisões melhores no orçamento e na gestão. (OECD, 2007, P. 3, TRADUÇÃO NOSSA).

Assim, a questão central do desempenho se mostra essencial, dada a necessidade e o anseio da população por melhores serviços e, no caso da saúde, por melhores condições de vida e saúde. Nesse sentido, têm sido frequentes os estudos que utilizam o desempenho como instrumento importante na avaliação da gestão dos sistemas e serviços de saúde (COSTA ET AL., 2015; MICLOS ET AL., 2015).

Este trabalho, portanto, parte de uma indagação central: em que medida é possível manter a relação entre o planejamento, o orçamento e o gasto em saúde e os efeitos sobre a melhoria da saúde da população? Tal questionamento nos coloca o desafio de utilizar uma ferramenta que responda à cumplicidade entre planejamento e orçamento, viabilizando uma política de saúde mais efetiva.

O presente estudo tem como objetivo avaliar o desempenho do gasto em saúde do município de São Bernardo do Campo, a partir de uma metodologia de desempenho do orçamento na saúde que busque relacionar o planejamento com os resultados da política de saúde alcançados. Assim, utiliza-se de uma metodologia baseada no instrumento Orçamento por Desempenho, que permite avaliar a relação entre as metas alcançadas no município de São Bernardo do Campo, os recursos envolvidos e os resultados das políticas de saúde sob a ótica do orçamento.

\section{Métodos}

Foram efetuados levantamentos e comparações dos dados secundários sobre gastos em saúde e indicadores de resultados em saúde do município de São Bernardo do Campo (SBC). A escolha desse município como estudo de caso deve-se ao fato de São Bernardo integrar a Região de Saúde do ABC - uma das mais organizadas e influentes regiões do estado de São Paulo - e por, desde 2009, ter se constituído no município 'modelo' das gestões do Partido dos Trabalhadores (PT), com inovações no processo de planejamento em saúde.

Destaca-se, ainda, que o município sempre apresentou um forte desenvolvimento econômico e de ampliação de sua extensão urbana, porém, com uma rede de serviços de saúde pública reconhecidamente desorganizada, numa lógica 'pré-SUS', ou seja, que, mesmo após a Constituição de 1988, Leis $n^{\circ}$ 
8.080/90 (BRASIL, 1990A) e $\mathrm{n}^{0} 8.142 / 90$ (BRASIL, 1990B), manteve estruturas que apenas no último quadriênio do período do estudo começaram a apresentar mudanças no sentido de fortalecimento do SUS em âmbito municipal (CHIORO, 2011; SANTOS JR., 2011).

O período de análise dos documentos e dados está compreendido entre 2006 e 2012, referente a duas gestões municipais (20062008 e 2009-2012). Importante destacar que o ano de 2005 não é considerado nesta análise, pois, para esse ano, o município de São Bernardo do Campo não disponibilizou o rol de indicadores via Pacto de Indicadores da Atenção Básica.

Essa caracterização da pesquisa possibilitou uma análise comparativa da gestão em saúde de diferentes grupos gestores.

O período torna-se ainda mais relevante por abranger a implantação do Pacto pela Saúde, instituído através da Portaria no 399, de 22 de fevereiro de 2006, que divulga e aprova as diretrizes do Pacto em 2006 (BRASIL, 2006). Além desse importante aspecto, o período contempla, ainda, a instituição de novos instrumentos, documentos e dinâmicas na gestão compartilhada do SUS, via Decreto $n^{\circ} 7.508 / 2011$ (BRASIL, 2011), que regulamentou a Lei no 8.080 (BRASIL, 1990A) ao explicitar conceitos, princípios e diretrizes do SUS, que passaram a exigir uma nova dinâmica na organização e na gestão do sistema de saúde.

A fim de assegurar adequado tratamento à indagação central deste estudo, foram utilizadas as informações orçamentárias do PPA, das LDOs e das LOAs, além das agregações das despesas conforme as subfunções indicadas pela Portaria no 42/1999, do Ministério do Planejamento, Orçamento e Gestão, tais como: atenção básica, assistência ambulatorial e hospitalar, produtos profiláticos e terapêuticos, vigilância sanitária, vigilância epidemiológica, alimentação e nutrição e outras subfunções. Essas informações foram relacionadas com a ferramenta que contribui para mensurar o melhor desempenho da ação pública em saúde, denominada
Performance Budget (PB), isto é, Orçamento por Desempenho (NOBREGA, 2011).

Segundo Nóbrega (2011), o PB constitui prática de entronizar nos orçamentos a avaliação de programas de governo, evidenciando quão efetivos podem ser os gastos públicos. Considerou-se tal tema relevante, uma vez que muitas áreas do governo carecem de uma adequada avaliação das prioridades e da mensuração de resultados.

Para a análise das informações e dos dados levantados, elaborou-se uma matriz de avaliação das ações que foram realizadas no campo da saúde pública pelo município escolhido, tendo em vista o impacto gerado segundo seu planejamento. Essa matriz contém os indicadores pactuados e selecionados em São Bernardo do Campo nos anos de análise destacados nos planos de saúde, nas pactuações regionais e, mais recentemente, presentes no Contrato Organizativo da Ação Pública da Saúde (Coap). Tais indicadores foram organizados segundo as categorias do PB: Recursos, Outputs, Eficiência e Outcomes.

Nóbrega (2011) define que, de acordo com um $\mathrm{PB}$, os resultados podem ser evidenciados através do impacto que o programa tem sobre os indivíduos, as estruturas sociais e o próprio governo. É necessária uma definição prévia dos resultados esperados para que, então, eles possam ser analisados segundo o esperado. Ainda, segundo o autor, para definição de produtos, algumas categorias são importantes:

Recursos: como número de professores, escolas construídas, livros distribuídos etc. Embora esses elementos não representem indicadores de performance, podem prover valiosas informações para controle de custos e para a elaboração de outros indicadores. Se o número de pessoas atendidas por determinado programa de governo está bem aquém daquilo que fora esperado, certamente medidas corretivas devem ser tomadas. 
Outputs: sua medida é fundamental porque representa o resultado imediato. É interessante observar que, na maioria das vezes, apenas há atenção na questão do resultado imediato, e, a partir daí, tiram-se conclusões.

Eficiência: em conceito econômico, significa output por unidade monetária; no entanto, quando avaliamos programas de governo, a resposta não pode ser tão simples. Os programas de governo devem atender a outros critérios além da mera questão quantitativa. Aspectos com efeitos redistributivos devem ser avaliados e muitas vezes passam ao largo de avaliações dos órgãos ou instâncias de controle. Assim, eficiência não é um conceito absoluto. Deve relacionar-se ao programa que está sendo avaliado.

Outcomes: representam um passo adiante na avaliação dos programas de governo. São indicadores mais amplos dos verdadeiros resultados, avaliando se o programa está tendo uma função transformadora na sociedade. São fundamentais para sinalizar ao governo se os objetivos estão sendo alcançados e quais medidas corretivas podem ser tomadas. (NOBREGA, 2011, P. 721).

Para a escolha dos indicadores a serem agrupados em cada uma das quatro dimensões do PB, deve-se destacar que, no decorrer dos anos, o número de indicadores pactuados entre os entes federados, e que deveriam ter acompanhamento ano a ano, variou. Assim, este estudo consolidou os indicadores que tiveram sua frequência em mais de três anos no período analisado (2006-2012), totalizando um conjunto de 33 indicadores.

Para a melhor compreensão da análise por desempenho, organizou-se uma tipologia dos indicadores e seus resultados por meio de cores distintas, apresentadas nas tabelas 1, 2, 3 e 4 do item Resultados. Para a meta alcançada, utilizamos a cor cinza claro, e para as metas não alcançadas, foi utilizada a cor cinza escuro.

Antes de iniciar as avaliações segundo categorias do $\mathrm{PB}$, destaca-se que no ano de 2006 não houve processo de pactuação e acompanhamento obrigatório para os entes federados, o que inviabiliza a obtenção dos dados com as metas estabelecidas naquele ano. Porém, este trabalho trouxe os dados de 2006 e os comparou com as metas que foram estabelecidas em 2007, a fim de assegurar uma análise mais ampla com relação à temporalidade dos resultados.

Para averiguação do alcance ou não das metas que foram propostas, ano a ano, as tabelas 1, 2, 3 e 4 tiveram suas colunas organizadas em Meta proposta (P1) e Resultado alcançado ( $\left.\mathrm{A}^{2}\right)$. Assim, comparou-se cada resultado obtido no ano com a sua respectiva meta proposta.

Os dados orçamentário-financeiros (despesas por subfunção) foram corrigidos segundo o Índice Geral de Preços - Disponibilidade Interna (IGP-DI), da Fundação Getúlio Vargas (FGV), com base em dezembro de 2012.

\section{Resultados e discussão}

Os resultados do presente trabalho mostram que a análise dos indicadores pactuados por São Bernardo do Campo e relacionados nos seus instrumentos de planejamento e nos relatórios de gestão, agregados segundo as categorias do Orçamento por Desempenho, foi satisfatória, apresentando uma melhora dos mesmos ao longo do período estudado.

Ao se iniciar a organização das categorias do PB, primeiramente, considera-se que, na categoria Recursos, os indicadores demonstraram como os recursos que estavam disponíveis à política de saúde em implementação foram utilizados para realizar ações em saúde, porém, que não indicaram, necessariamente, a realização de processos de trabalho, resultados imediatos ou resultados de longo prazo. 
A tabela 1 apresenta que tanto o primeiro quanto o segundo indicador - 'Média anual de consultas médias por habitante nas especialidades básicas' e 'Cobertura da primeira consulta odontológica programática' - não foram acompanhados nos últimos três anos de análise e que não tiveram suas metas atingidas nos anos anteriores.

Tabela 1. Relação de indicadores estabelecidos para a categoria Recursos do Orçamento por Desempenho segundo despesa por subfunção em São Bernardo do Campo, de 2006 a 2012

\begin{tabular}{|c|c|c|c|c|c|c|c|c|c|c|c|c|c|c|}
\hline \multirow[b]{2}{*}{ Subfunção } & \multirow[b]{2}{*}{ Indicador } & \multirow{2}{*}{$\frac{2006}{A^{2}}$} & \multicolumn{2}{|c|}{2007} & \multicolumn{2}{|c|}{2008} & \multicolumn{2}{|c|}{2009} & \multicolumn{2}{|c|}{2010} & \multicolumn{2}{|c|}{2011} & \multicolumn{2}{|c|}{2012} \\
\hline & & & $\mathbf{P}^{1}$ & $A^{2}$ & $\mathbf{P} 1$ & $A^{2}$ & $\mathbf{P}^{1}$ & $A^{2}$ & $\mathbf{P}^{1}$ & $A^{2}$ & $\mathbf{P}^{1}$ & $A^{2}$ & $\mathbf{P}^{1}$ & $A^{2}$ \\
\hline $\begin{array}{l}301 \text { - } \\
\text { Atenção } \\
\text { Básica }\end{array}$ & $\begin{array}{l}\text { Média anual } \\
\text { de consultas } \\
\text { médicas por } \\
\text { habitante nas } \\
\text { especialidades } \\
\text { básicas }\end{array}$ & 1,6 & 1,7 & 1,66 & 1,7 & 0,87 & 1,7 & - & - & - & - & - & - & - \\
\hline $\begin{array}{l}301 \text { - } \\
\text { Atenção } \\
\text { Básica }\end{array}$ & $\begin{array}{l}\text { Cobertura } \\
\text { da primeira } \\
\text { consulta odon- } \\
\text { tológica pro- } \\
\text { gramática }\end{array}$ & 4,8 & 11 & 4,1 & 11,5 & 6,22 & 11,5 & 5,65 & - & - & - & - & - & - \\
\hline $\begin{array}{l}301 \text { - } \\
\text { Atenção } \\
\text { Básica }\end{array}$ & $\begin{array}{l}\text { Proporção da } \\
\text { população } \\
\text { cadastrada pela } \\
\text { Estratégia Saú- } \\
\text { de da Família }\end{array}$ & 7,43 & 7,43 & 6,47 & 7,43 & 5,33 & 7,43 & 5,7 & 18 & 8,95 & 30 & 36,8 & 50 & 47,77 \\
\hline $\begin{array}{l}301 \text { - } \\
\text { Atenção } \\
\text { Básica }\end{array}$ & $\begin{array}{l}\text { Percentual de } \\
\text { famílias com } \\
\text { perfil saúde } \\
\text { beneficiárias } \\
\text { do Programa } \\
\text { Bolsa Família } \\
\text { acompanhadas } \\
\text { pela atenção } \\
\text { básica }\end{array}$ & - & - & - & 45,8 & 42,77 & 45,8 & 46,49 & 47 & 31,07 & 48 & 53,78 & 58 & 61,95 \\
\hline $\begin{array}{l}305 \text { - Vigi- } \\
\text { lância Epide- } \\
\text { miológica }\end{array}$ & $\begin{array}{l}\text { Cobertura } \\
\text { vacinal por } \\
\text { tetravalente em } \\
\text { menores de um } \\
\text { ano de idade }\end{array}$ & 95,59 & 95 & 97,5 & 95 & 99 & 95 & 97,5 & 95 & 75,94 & 95 & 97,01 & 95 & 93,7 \\
\hline $\begin{array}{l}\text { Outras } \\
\text { subfunções }\end{array}$ & $\begin{array}{l}\text { Taxa de cober- } \\
\text { tura Caps por } \\
100 \text { mil habi- } \\
\text { tantes }\end{array}$ & - & - & - & 0,26 & 0 & 0,26 & 0,24 & - & - & 0,92 & 0,59 & 1 & 1,03 \\
\hline $\begin{array}{l}\text { Outras } \\
\text { subfunções }\end{array}$ & $\begin{array}{l}\text { Índice de con- } \\
\text { tratualização } \\
\text { de unidades } \\
\text { conveniadas ao } \\
\text { SUS }\end{array}$ & 100 & 100 & 100 & 100 & 100 & - & - & - & - & - & - & 75 & 75 \\
\hline
\end{tabular}

Fonte: Sispacto (Sistema de Pactuação dos Indicadores).

1 Meta proposta.

2 Resultado alcançado.

(-) Sem registro 
Entre os cinco demais indicadores, os melhores resultados ficaram com o indicador 'Cobertura vacinal por tetravalente em menores de um ano de idade', que, apenas em 2010, teve resultado abaixo da meta estabelecida.

Importante destacar que, para o indicador 'Proporção da população cadastrada pela estratégia saúde da família', somente após 2009, as metas estabelecidas se tornaram mais 'ousadas', com a proposição de aumento de cobertura, e que, ano a ano, foi melhorando (2011 e 2012). Fato também encontrado para o indicador de 'Taxa de cobertura Centro de Apoio Psicossocial (Caps) por 100 mil habitantes' para o ano de 2012 (tabela 1).

$\mathrm{O}$ 'Índice de contratualização de unidades conveniadas ao SUS' teve $100 \%$ de alcance das metas entre 2006 e 2008, porém, nos anos de 2009, 2010 e 2011, esse indicador não esteve presente nas pactuações do município.

Nota-se, também, que a categoria Resultados do PB teve melhor desempenho, isto é, alcance de metas dos indicadores, no segundo quadriênio do período analisado, sendo 7 bons resultados no $1^{\circ}$ quadriênio da gestão (2005 a 2008), e 10 no $2^{\circ}$ quadriênio (2009 a 2012).

Entre 2006 e 2012, o indicador 'Cobertura vacinal por tetravalente em menores de um ano de idade' (indicador associado à vigilância epidemiológica) obteve um excelente desempenho, na medida em que os resultados alcançados foram superiores às metas propostas. Pode-se inferir que tal situação foi facilitada pelos constantes e crescentes recursos despendidos com a subfunção 'vigilância epidemiológica', que obteve um acréscimo de seus recursos de $3.616 \%$, passando de $\mathrm{R} \$ 281,0$ mil para $\mathrm{R} \$ 10,4$ milhões, respectivamente.

Uma relação importante é feita considerando as 'Despesas liquidadas e inscritas em restos a pagar segundo a subfunção' atenção básica. A partir de 2010, as despesas com essa subfunção são retomadas e têm seus valores em crescimento, passando de R $\$ 90$ milhões, em 2010, para R \$ 111 milhões, em 2011, e atingindo $\mathrm{R} \$ 141$ milhões, em 2012. Essa evolução pode ter garantido suficiência de recursos para o cumprimento das metas para os indicadores sobre a 'Proporção da população cadastrada pela Estratégia Saúde da Família’ e o 'Percentual de famílias com perfil saúde beneficiárias do Programa Bolsa Família acompanhadas pela atenção básica', conforme indica a tabela 1 .

Para a análise dos indicadores na categoria Outputs, considerou-se que os indicadores relacionados deviam demonstrar alguns resultados imediatos e que o município possivelmente os alcançou mediante ações em saúde realizadas, porém, não necessariamente indicaram real impacto em melhores condições de vida da população. Importante citar que esses indicadores teriam e têm grande relevância de uso nas LDOs, uma vez que essas representam e devem conter as avaliações das metas ano a ano, diferentemente dos PPAs, que olham os resultados a cada quadriênio.

De forma geral, de acordo com a tabela 2, das 40 avaliações realizadas para os 7 indicadores relacionados ao longo dos anos em estudo, 15 não superaram as metas. $\mathrm{O}$ indicador de 'Taxa de cura de casos novos de tuberculose bacilífera' foi o que apresentou os piores resultados durante os anos, tendo sua meta superada apenas em 2006. 
Tabela 2. Relação de indicadores estabelecidos para a categoria Outputs do Orçamento por Desempenho segundo despesas por subfunção em São Bernardo do Campo, de 2006 a 2012

\begin{tabular}{|c|c|c|c|c|c|c|c|c|c|c|c|c|c|c|}
\hline \multirow[b]{2}{*}{ Subfunção } & \multirow[b]{2}{*}{ Indicador } & \multirow{2}{*}{$\frac{2006}{A^{2}}$} & \multicolumn{2}{|c|}{2007} & \multicolumn{2}{|c|}{2008} & \multicolumn{2}{|c|}{2009} & \multicolumn{2}{|c|}{2010} & \multicolumn{2}{|c|}{2011} & \multicolumn{2}{|c|}{2012} \\
\hline & & & $P^{1}$ & $A^{2}$ & $P^{1}$ & $A^{2}$ & $\mathbf{P} 1$ & $A^{2}$ & $\mathbf{P}^{1}$ & $A^{2}$ & $\mathbf{P} 1$ & $A^{2}$ & $\mathbf{P} 1$ & $A^{2}$ \\
\hline $\begin{array}{l}301 \text { - } \\
\text { Atenção } \\
\text { Básica }\end{array}$ & $\begin{array}{l}\text { Taxa de in- } \\
\text { ternação } \\
\text { por diabetes } \\
\text { mellitus e suas } \\
\text { complicações } \\
\text { na população } \\
\text { de } 30 \text { anos e } \\
\text { mais }\end{array}$ & 1,52 & 1,3 & 11,4 & 9,7 & 4,08 & 9,7 & 5,4 & 3,6 & 1,51 & 3 & 2,16 & - & - \\
\hline $\begin{array}{l}302 \text { - } \\
\text { Assistência } \\
\text { Hospitalar e } \\
\text { Ambulatorial }\end{array}$ & $\begin{array}{l}\text { Taxa de inter- } \\
\text { nações por } \\
\text { Acidente Vas- } \\
\text { cular Cerebral } \\
(A \vee C)\end{array}$ & 21,5 & 21,5 & 20,7 & 20,7 & 19,7 & 20,7 & 20,1 & 7,2 & 4,79 & 7 & 6,79 & - & - \\
\hline $\begin{array}{l}302 \text { - } \\
\text { Assistência } \\
\text { Hospitalar e } \\
\text { Ambulatorial }\end{array}$ & $\begin{array}{l}\text { Taxa de cura } \\
\text { de hanseníase } \\
\text { nos anos das } \\
\text { coortes }\end{array}$ & 86,1 & 80 & 100 & 90 & 85 & 90 & 91,3 & 90 & 79,3 & 90 & 100 & 89 & 95 \\
\hline $\begin{array}{l}302 \text { - } \\
\text { Assistência } \\
\text { Hospitalar e } \\
\text { Ambulatorial }\end{array}$ & $\begin{array}{l}\text { Taxa de cura } \\
\text { de casos novos } \\
\text { de tuberculose } \\
\text { bacilífera }\end{array}$ & 87,6 & 85 & 81,8 & 85 & 81,8 & 85 & 75,7 & 85 & 19,8 & 85 & 83 & 85 & 42,6 \\
\hline $\begin{array}{l}302 \text { - } \\
\text { Assistência } \\
\text { Hospitalar e } \\
\text { Ambulatorial }\end{array}$ & $\begin{array}{l}\text { Proporção de } \\
\text { partos cesáreos }\end{array}$ & 57,8 & 46,2 & 36,1 & 38,4 & 33,8 & 38,4 & 35,2 & - & - & - & - & 43 & 37,1 \\
\hline $\begin{array}{l}302 \text { - } \\
\text { Assistência } \\
\text { Hospitalar e } \\
\text { Ambulatorial }\end{array}$ & $\begin{array}{l}\text { Taxa de in- } \\
\text { ternação } \\
\text { hospitalar de } \\
\text { pessoas idosas } \\
\text { por fratura de } \\
\text { fêmur }\end{array}$ & - & - & - & 13,7 & 12,1 & 13,7 & 14,4 & 14,1 & 12 & 13,9 & 14,4 & 14 & 14 \\
\hline $\begin{array}{l}306 \text { - } \\
\text { Alimentação } \\
\text { e Nutrição }\end{array}$ & $\begin{array}{l}\text { Percentual de } \\
\text { crianças me- } \\
\text { nores de cinco } \\
\text { anos com baixo } \\
\text { peso para idade }\end{array}$ & - & - & - & 3 & 3,5 & 3 & 2,63 & 2,6 & 1,75 & 3 & 2,5 & - & - \\
\hline
\end{tabular}

Fonte: Sispacto (Sistema de Pactuação dos Indicadores).

1 Meta proposta.

2 Resultado alcançado.

(-) Sem registro. 
As taxas de internação por diabetes mellitus e por Acidente Vascular Cerebral (AVC) tiveram resultados que superaram as metas de 2008 a 2011, casos parecidos com o de 'Percentual de crianças menores de cinco com baixo peso para a idade'.

Destaca-se aqui o indicador 'Proporção de partos cesáreos', pois representa uma mudança real em toda uma rede de cuidado em saúde que se inicia na orientação e na indicação ao parto normal, desde uma visita domiciliar realizada por uma equipe de saúde até a organização e o trabalho, com uma educação em saúde que leve as equipes dos hospitais a realizar mais partos normais em vez dos cesáreos. Trata-se de um indicador que representa uma mudança em toda uma forma de cuidado em diferentes pontos da rede de serviços de saúde.

Tem-se, então, que para a categoria Outputs houve o cumprimento de metas por 9 vezes, considerando todos os indicadores. Situação diferente foi encontrada no $2^{\circ}$ quadriênio, em que o alcance das metas ocorreu 16 vezes.

Ao relacionar essa categoria com as 'Despesas liquidadas e inscritas em restos a pagar segundo sua subfunção', a partir de 2010, as despesas com assistência hospitalar e ambulatorial apresentam crescimento, passando de R\$ 451 milhões, em 2010, para R\$ 506 milhões, em 2011, e alcançando R\$ 557 milhões, em
2012. Esse aumento da despesa pode ter garantido o cumprimento das metas para os indicadores sobre 'Taxa de internações por Acidente Vascular Cerebral (AVC)', 'Taxa de cura de hanseníase nos anos das coortes', 'Proporção de partos cesáreos' e 'Taxa de internação hospitalar de pessoas idosas por fratura de fêmur', conforme indicado na tabela 2.

Para a categoria Eficiência, procurou-se associar o conceito de Nóbrega (2011, P. 694) - que indica a necessidade de se avaliar a eficiência "não como um conceito absoluto, devendo relacionar-se ao programa que está sendo avaliado" - com o conceito de eficiência, elaborado pelo Tribunal de Contas da União (TCU) (BRASIL, 2000, P. 15), enquanto "dimensão que se refere ao esforço do processo de transformação de insumos em produtos". Assim, para além da visão clássica econômica que, muitas vezes, atribui à eficiência a noção de custo-benefício (melhor resultado com o menor recurso empregado), trabalhou-se com a definição de eficiência que se refere à garantia de realização do processo de trabalho/cuidado que, não necessariamente, acarretou resultados imediatos ou em longo prazo.

Quando analisada a tabela 3, identifica-se que os três primeiros indicadores tiveram, nos primeiros anos, resultados aquém das metas propostas, com melhora no desempenho a partir de 2011.

Tabela 3. Relação de indicadores estabelecidos para a categoria Eficiência do Orçamento por Desempenho segundo despesas por subfunção em São Bernardo do Campo, de 2006 a 2012

\begin{tabular}{|c|c|c|c|c|c|c|c|c|c|c|c|c|c|c|}
\hline \multirow[b]{2}{*}{ Subfunção } & \multirow[b]{2}{*}{ Indicador } & \multirow{2}{*}{$\begin{array}{c}2006 \\
A^{2}\end{array}$} & \multicolumn{2}{|c|}{2007} & \multicolumn{2}{|c|}{2008} & \multicolumn{2}{|c|}{2009} & \multicolumn{2}{|c|}{2010} & \multicolumn{2}{|c|}{2011} & \multicolumn{2}{|c|}{2012} \\
\hline & & & $\mathbf{P}^{1}$ & $A^{2}$ & $\mathbf{P}^{1}$ & $A^{2}$ & $\mathbf{P}^{1}$ & $A^{2}$ & $\mathbf{P}^{1}$ & $A^{2}$ & $\mathbf{P}^{1}$ & $A^{2}$ & $\mathbf{P}^{1}$ & $A^{2}$ \\
\hline $\begin{array}{l}301 \text { - } \\
\text { Atenção } \\
\text { Básica }\end{array}$ & $\begin{array}{l}\text { Razão entre } \\
\text { exames pre- } \\
\text { ventivos do } \\
\text { câncer do colo } \\
\text { do útero em } \\
\text { mulheres de } \\
25 \text { a } 59 \text { anos } \\
\text { e a população } \\
\text { feminina nesta } \\
\text { faixa etária }\end{array}$ & 0,14 & 0,2 & 0,11 & 0,2 & 0,06 & 0,2 & 0,1 & 0,15 & 0,04 & 0,18 & 0,18 & 0,4 & 0,5 \\
\hline
\end{tabular}




\section{Tabela 3. (cont.)}

\begin{tabular}{|c|c|c|c|c|c|c|c|c|c|c|c|c|c|c|}
\hline $\begin{array}{l}301 \text { - } \\
\text { Atenção } \\
\text { Básica }\end{array}$ & $\begin{array}{l}\text { Proporção de } \\
\text { nascidos vivos } \\
\text { de mães com } \\
7 \text { ou mais } \\
\text { consultas de } \\
\text { pré-natal }\end{array}$ & 80,4 & 80 & 77,8 & 75 & 80,5 & 75 & 81 & 80,5 & - & 80,8 & 81,3 & 80 & 80,4 \\
\hline $\begin{array}{l}301 \text { - } \\
\text { Atenção } \\
\text { Básica }\end{array}$ & $\begin{array}{l}\text { Média anual } \\
\text { da ação cole- } \\
\text { tiva escovação } \\
\text { dental supervi- } \\
\text { sionada }\end{array}$ & - & 3,5 & 1,3 & 3,5 & 1,49 & 3,5 & 0,52 & - & - & 3,11 & 3,17 & 3,2 & 2,09 \\
\hline $\begin{array}{l}301 \text { - } \\
\text { Atenção } \\
\text { Básica }\end{array}$ & $\begin{array}{l}\text { Média mensal } \\
\text { de visitas do- } \\
\text { miciliares por } \\
\text { família }\end{array}$ & - & 0 & 0,78 & 1 & 0,77 & 1 & 0,66 & - & - & - & - & - & - \\
\hline $\begin{array}{l}302 \text { - } \\
\text { Assistência } \\
\text { Hospitalar e } \\
\text { Ambulatorial }\end{array}$ & $\begin{array}{l}\text { Taxa de notifi- } \\
\text { cação de casos } \\
\text { de Paralisia } \\
\text { Flácida Agu- } \\
\text { da (PFA) em } \\
\text { menores de } 15 \\
\text { anos }\end{array}$ & 1,44 & 1 & 0 & 2 & 2 & 2 & 0 & - & - & - & - & - & - \\
\hline $\begin{array}{l}302 \text { - } \\
\text { Assistência } \\
\text { Hospitalar e } \\
\text { Ambulatorial }\end{array}$ & $\begin{array}{l}\text { Percentual de } \\
\text { seguimento/ } \\
\text { tratamento } \\
\text { informado de } \\
\text { mulheres com } \\
\text { diagnóstico de } \\
\text { lesões intrae- } \\
\text { piteliais de alto } \\
\text { grau do colo do } \\
\text { útero }\end{array}$ & - & - & - & 100 & 3,69 & 100 & 88,6 & 80 & 59,8 & 85 & 87,7 & 86 & 82,1 \\
\hline $\begin{array}{l}302 \text { - } \\
\text { Assistência } \\
\text { Hospitalar e } \\
\text { Ambulatorial }\end{array}$ & $\begin{array}{l}\text { Razão entre } \\
\text { mamografias } \\
\text { realizadas nas } \\
\text { mulheres de } \\
50 \text { a } 69 \text { anos } \\
\text { e a população } \\
\text { feminina nesta } \\
\text { faixa etária, em } \\
\text { determinado } \\
\text { local e ano. }\end{array}$ & - & - & - & - & - & - & - & 0,18 & 0,15 & 0,2 & 0,21 & 0,4 & 0,42 \\
\hline $\begin{array}{l}303 \text { - } \\
\text { Suporte } \\
\text { Profilático e } \\
\text { Terapêutico }\end{array}$ & $\begin{array}{l}\text { Proporção } \\
\text { de casos de } \\
\text { hepatites b e } \\
\text { c confirmados } \\
\text { por sorologia }\end{array}$ & - & - & - & 95 & 85 & 95 & 97 & 95 & 95,5 & 95 & 98,8 & - & - \\
\hline $\begin{array}{l}305 \text { - Vigi- } \\
\text { lância Epide- } \\
\text { miológica }\end{array}$ & $\begin{array}{l}\text { Proporção de } \\
\text { óbitos de mu- } \\
\text { Iheres em idade } \\
\text { fértil investi- } \\
\text { gados }\end{array}$ & 93,9 & 75 & 94 & 75 & 77,2 & 75 & 75 & 80 & - & 90 & 96,8 & 95 & 80 \\
\hline
\end{tabular}




\begin{tabular}{|c|c|c|c|c|c|c|c|c|c|c|c|c|c|c|}
\hline $\begin{array}{l}305 \text { - Vigi- } \\
\text { lância Epide- } \\
\text { miológica }\end{array}$ & $\begin{array}{l}\text { Proporção } \\
\text { de doenças } \\
\text { exantemáticas } \\
\text { investigadas } \\
\text { oportunamente }\end{array}$ & 100 & 80 & 96,4 & 80 & 88,5 & 80 & 93,9 & - & - & - & - & - & - \\
\hline $\begin{array}{l}305 \text { - Vigi- } \\
\text { lância Epide- } \\
\text { miológica }\end{array}$ & $\begin{array}{l}\text { Proporção de } \\
\text { óbitos não fe- } \\
\text { tais informados } \\
\text { ao SIM com } \\
\text { causa básica } \\
\text { definida }\end{array}$ & 99,3 & 95 & 99,6 & 95 & 99,1 & 95 & 99 & 95 & - & 95 & 99,2 & 95 & 81 \\
\hline $\begin{array}{l}305 \text { - Vigi- } \\
\text { lância Epide- } \\
\text { miológica }\end{array}$ & $\begin{array}{l}\text { Números de } \\
\text { casos de sífilis } \\
\text { congênita } \\
\text { notificados }\end{array}$ & 10 & 18 & 4 & 3 & - & - & - & 7 & 10 & 11 & 19 & 28 & 21 \\
\hline $\begin{array}{l}305 \text { - Vigi- } \\
\text { lância Epide- } \\
\text { miológica }\end{array}$ & $\begin{array}{l}\text { Proporção de } \\
\text { investigação de } \\
\text { óbitos infantis }\end{array}$ & - & - & - & 30 & 53,3 & 30 & 65,9 & - & - & - & - & 95 & 87 \\
\hline $\begin{array}{l}305 \text { - Vigi- } \\
\text { lância Epide- } \\
\text { miológica }\end{array}$ & $\begin{array}{l}\text { Proporção } \\
\text { de casos de } \\
\text { Doenças de } \\
\text { Notificação } \\
\text { Compulsória } \\
\text { (DNC) encer- } \\
\text { rados oportu- } \\
\text { namente após } \\
\text { notificação }\end{array}$ & - & - & - & 80 & 91,2 & 80 & 89 & 80 & 90,3 & 88 & 95,9 & 90 & 97,9 \\
\hline
\end{tabular}

Fonte: Sispacto (Sistema de Pactuação dos Indicadores).

${ }^{1}$ Meta proposta.

2 Resultado alcançado.

(-) Sem registro.

Os indicadores 'Média mensal de visitas domiciliares por família' e 'Taxa de notificação de casos de Paralisia Flácida Aguda (PFA) em menores de 15 anos' não foram considerados nas pactuações após 2009, tendo o primeiro indicador apenas uma vez a meta cumprida (2007), e o segundo, metas alcançadas em todos os anos.

Quando analisado o indicador 'Percentual de seguimento/tratamento informado de mulheres com diagnóstico de lesões intraepiteliais de alto grau do colo do útero', notou-se que apenas em 2011 a meta proposta foi alcançada, tendo por quatro anos resultados abaixo do esperado.

Já para a 'Razão entre mamografias realizadas nas mulheres de 50 a 69 anos e a população feminina nesta faixa etária, em determinado local e ano', foi possível observar o uso do indicador a partir de 2010, não obtendo um bom resultado nesse ano, porém, alcançando as metas nos dois anos seguintes (2011 e 2012).

A 'Proporção de casos de hepatites B e C confirmados por sorologia' teve alcance de metas a partir do ano de 2009, porém, não foi considerado como indicador para avaliação em 2012 (tabela 3).

$\mathrm{O}$ indicador 'Proporção de doenças exantemáticas investigadas oportunamente’ teve bons resultados nos anos em que foi utilizado (alcance das metas propostas), porém, a partir de 2010, não foi mais utilizado.

A respeito dos indicadores 'Proporção de óbitos de mulheres em idade fértil investigados', 'Proporção de óbitos não fetais informados ao SIM (Sistema de Informação sobre 
Mortalidade) com causa básica definida', 'Números de casos de sífilis congênita notificados' e 'Proporção de investigação de óbitos infantis', houve uso em quase todos os anos de análise, porém, em 2011 e 2012, obteve-se resultados aquém das metas propostas (tabela 3).

Observa-se que o indicador 'Proporção de casos de Doenças de Notificação Compulsória (DNC) encerrados oportunamente após notificação' foi utilizado em praticamente todos os anos, com bons resultados em todos eles.

Ao se considerar o quadro total para a categoria Eficiência, nota-se que o $1^{\circ}$ quadriênio obteve maior número de metas alcançadas, 18 , ao passo que o $2^{\circ}$ quadriênio atingiu 15 .

Uma relação importante, ainda considerando a categoria Eficiência, é feita considerando as 'Despesas liquidadas e inscritas em restos a pagar segundo a subfunção'. Como mencionado na análise da categoria resultados, a partir de 2010, as despesas com a subfunção 'atenção básica' foram crescentes. Tal fato pode ter contribuído para o cumprimento das metas para os indicadores: 'Razão entre exames preventivos do câncer do colo do útero em mulheres de 25 a 59 anos e a população feminina nesta faixa etária', 'Proporção de nascidos vivos de mães com 7 ou mais consultas de pré-natal' e 'Média anual da ação coletiva escovação dental supervisionada' (tabela 3).

Para a categoria Outcomes organizaram-se indicadores que representam um passo adiante na avaliação dos programas de governo, sendo possível a análise de resultados em médio e longo prazos, isto é, resultados mais efetivos junto à população, possibilitando uma avaliação a respeito de os programas estarem ou não cumprindo uma função transformadora na sociedade.

A tabela 4 mostra, positivamente, que o indicador 'Taxa de letalidade por febre hemorrágica de dengue' foi mantido como indicador a partir de 2008 e que teve bons resultados em todos os demais anos.

Tabela 4. Relação de indicadores estabelecidos para a categoria Outcomes do Orçamento por Desempenho segundo despesas por subfunção de São Bernardo do Campo, de 2006 a 2012

\begin{tabular}{|c|c|c|c|c|c|c|c|c|c|c|c|c|c|c|}
\hline \multirow[b]{2}{*}{ Subfunção } & \multirow[b]{2}{*}{ Indicador } & \multirow{2}{*}{$\frac{2006}{A^{2}}$} & \multicolumn{2}{|c|}{2007} & \multicolumn{2}{|c|}{2008} & \multicolumn{2}{|c|}{2009} & \multicolumn{2}{|c|}{2010} & \multicolumn{2}{|c|}{2011} & \multicolumn{2}{|c|}{2012} \\
\hline & & & $\mathbf{P} 1$ & $A^{2}$ & $\mathbf{P} 1$ & $A^{2}$ & $\mathbf{P} 1$ & $A^{2}$ & $\mathbf{P} 1$ & $A^{2}$ & $\mathbf{P} 1$ & $A^{2}$ & $\mathbf{P} 1$ & $A^{2}$ \\
\hline $\begin{array}{l}302 \text { - } \\
\text { Assistência } \\
\text { Hospitalar e } \\
\text { Ambulatorial }\end{array}$ & $\begin{array}{l}\text { Taxa de letali- } \\
\text { dade por febre } \\
\text { hemorrágica de } \\
\text { dengue }\end{array}$ & - & - & - & 0 & 0 & 0 & 0 & 0 & 0 & 0 & 0 & 0 & 0 \\
\hline $\begin{array}{l}303 \text { - } \\
\text { Suporte } \\
\text { Profilático e } \\
\text { Terapêutico }\end{array}$ & $\begin{array}{l}\text { Taxa de morta- } \\
\text { lidade por Aids }\end{array}$ & 5,58 & 6 & 0 & 3,1 & 0 & 3,1 & 0 & 1 & 0 & 1,2 & 3,67 & 3,3 & 2 \\
\hline $\begin{array}{l}\text { Outras } \\
\text { subfunções }\end{array}$ & $\begin{array}{l}\text { Coeficiente de } \\
\text { mortalidade } \\
\text { infantil }\end{array}$ & 12,5 & 12,5 & 12,5 & - & - & - & - & - & - & 12 & 10 & 9,9 & 9,4 \\
\hline $\begin{array}{l}\text { Outras } \\
\text { subfunções }\end{array}$ & $\begin{array}{l}\text { Coeficiente de } \\
\text { mortalidade } \\
\text { neonatal }\end{array}$ & 7,64 & 7,25 & 8,08 & 7,7 & 7,8 & 7,7 & 6,9 & - & - & 7,45 & 6,29 & - & - \\
\hline $\begin{array}{l}\text { Outras } \\
\text { subfunções }\end{array}$ & $\begin{array}{l}\text { Coeficiente de } \\
\text { mortalidade } \\
\text { neonatal tardia }\end{array}$ & 2,64 & 2 & 5,04 & 4,7 & 4,2 & 4,7 & 6 & - & - & 4,54 & 3,74 & - & - \\
\hline \multicolumn{15}{|c|}{ Fonte: Sispacto (Sistema de Pactuação dos Indicadores). } \\
\hline \multicolumn{15}{|c|}{${ }^{1}$ Meta proposta. } \\
\hline \multicolumn{15}{|c|}{2 Resultado alcançado. } \\
\hline
\end{tabular}


Para o indicador 'Taxa de mortalidade por Aids' por 100 mil habitantes, nota-se o cumprimento das metas em quase todos os anos analisados, com exceção de 2011. Importante notar que, a partir de 2010, as metas propostas foram reduzidas para 1,0 , porém, o indicador voltou ao patamar de 3,0 em 2012, assim como nos anos que antecederam 2010.

Os três últimos indicadores - 'Coeficiente de mortalidade infantil', 'Coeficiente de mortalidade neonatal' e 'Coeficiente de mortalidade neonatal tardia' - guardam uma relação entre si por se tratarem de diferentes focos sobre a mortalidade infantil. $\mathrm{O}$ primeiro abrange uma cobertura mais geral (menores de um ano de idade), o segundo é específico para os bebês neonatos (bebês de 0 a 28 dias de vida completos), e o último refere-se aos neonatos tardios (bebês de 7 a 27 dias de vida completos) (tabela 4).

o 'Coeficiente de mortalidade infantil' compõe o Índice de Desenvolvimento Humano (IDH) na sua dimensão Longevidade (IDH-L) e é um dos principais coeficientes que as gestões públicas em saúde têm perseguido, esforçando-se para alcançar melhores resultados. No caso do município estudado, os dois primeiros anos apresentaram resultados abaixo da meta, com melhora do quadro a partir de 2011 e 2012, superando a meta que também foi mais 'ousada' (reduzida) nesse último ano.

Os 'Coeficientes de mortalidade neonatal e neonatal tardia' tiveram, na maioria dos quatro primeiros anos, um desempenho ruim, vindo a apresentar melhores resultados no ano de 2011.

A análise geral da tabela 4 demonstra, de forma unânime, que, no $1^{\circ}$ quadriênio, obtiveram-se 5 resultados positivos (metas alcançadas), ao passo que, no $2^{\circ}$ quadriênio, houve 12.

Uma relação importante é feita considerando-se as 'Despesas liquidadas e inscritas em restos a pagar segundo subfunção', onde, a partir de 2010, as despesas com as 'outras subfunções' são claramente aumentadas (médias de R 7.281.295,49 no $1^{\circ}$ quadriênio e R \$ 41.371.293,68 no $2^{\circ}$ quadriênio), o que pode ter garantido suficiência de recursos para o cumprimento das metas para os indicadores sobre 'Coeficiente de mortalidade infantil', 'Coeficiente de mortalidade neonatal' e 'Coeficiente de mortalidade neonatal tardia', como pôde ser visto na tabela 4.

Como este trabalho tem um foco essencial sobre o desempenho das políticas públicas em saúde e sua relação com os instrumentos orçamentários, elaborou-se a tabela 5, que demonstra um quadro geral da quantidade de metas alcançadas em cada um dos quadriênios em que os indicadores foram utilizados e, possivelmente, acompanhados. Segundo a tabela 5, nota-se o predomínio de melhores resultados - metas cumpridas - no $2^{\circ}$ quadriênio para as categorias Recursos, Outputs e Outcomes, ficando abaixo do $1^{\circ}$ quadriênio apenas na categoria Eficiência. Na média, o primeiro período atingiu $50 \%$ de sucesso no alcance de metas propostas, ao passo que, no segundo período, $63 \%$ das metas propostas foram cumpridas.

Tabela 5. Quadro geral das metas alcançadas segundo categoria do PB e quadriênio ( $1^{\circ}$ e $2^{\circ}$ ) em São Bernardo do Campo, de 2006 a 2012

\begin{tabular}{lcccccccc}
\hline & \multicolumn{2}{c}{ Recursos } & \multicolumn{2}{c}{ Outputs } & \multicolumn{2}{c}{ Eficiência } & \multicolumn{2}{c}{ Outcomes } \\
\hline Quadriênio & $\mathbf{1}$ & $\mathbf{2 0}$ & $\mathbf{1}$ & $\mathbf{2}$ & $\mathbf{1}$ & $\mathbf{2}$ & $\mathbf{1}$ & $\mathbf{2}$ \\
\hline Avaliações (a) & 17 & 17 & 17 & 23 & 28 & 38 & 12 & 14 \\
Metas cumpridas (b) & 7 & 10 & 9 & 16 & 18 & 15 & 5 & 12 \\
Relação percentual (b/a) & $41 \%$ & $59 \%$ & $53 \%$ & $70 \%$ & $64 \%$ & $39 \%$ & $42 \%$ & $86 \%$ \\
\hline
\end{tabular}

Fonte: Elaboração própria. 
Pode-se inferir que o grupo gestor, presente no primeiro quadriênio do período analisado, teve uma preocupação com a garantia de bons resultados, focando-se mais nos processos de trabalho/cuidado que eram realizados e não necessariamente nos resultados que poderiam alcançar (Outputs e Outcomes) (tabela 5).

Já o segundo grupo gestor, além de obter maior êxito no alcance das metas propostas, esteve focado, muito provavelmente, em alcançar resultados com as políticas de saúde que estavam implantando e/ou desenvolvendo. Por sua vez, então, cabe mencionar que os resultados obtidos no segundo quadriênio foram também alcançados pelo esforço das ações empreendidas no primeiro.

Com o aprofundamento da investigação dos instrumentos orçamentários (PPAs, LDOs e LOAs), foi possível perceber que houve uma evolução histórica do uso e da organização daqueles instrumentos, num sentido de considerar o desempenho/resultado como ponto fundamental, surgindo, então, o ainda vigente orçamento-programático.

Por essa lógica, o Orçamento por Desempenho, já praticado, minimamente, em vários países que compõem a OECD, seria um passo adiante na qualificação do orçamento-programa, uma vez que os indicadores estruturados segundo categorias (Recursos, Outputs, Eficiência, Outcomes) ajudam e necessitam que os instrumentos orçamentários estejam bem organizados e propostos.

Os resultados indicaram que, aproximadamente, $40 \%$ dos países que compõem a OECD iniciaram o uso de medições de resultados há pelo menos 10 ou mais anos, sendo que apenas 7,4\% dos países encontravam-se em fase de desenvolvimento dessa estratégia.

Quando perguntados sobre que tipos de informações para o desempenho devem ser produzidos para avaliar a ação do governo, observou-se que o desenvolvimento de informações de desempenho é uma tendência generalizada em quase três quartos dos países da OECD. Os países têm adotado diferentes abordagens para a avaliação do desempenho, porém, apresentaram as formas de avaliação e de medições de desempenho em quantidades iguais.

Observou-se que, no tocante ao tempo em que foi iniciado o último esforço do governo para medir o seu desempenho, os países não têm uma uniformidade no período, apresentando constantes mudança e evolução. Ao longo dos últimos cinco anos, $75 \%$ dos países da OECD introduziram uma nova iniciativa.

Pode-se vislumbrar, por exemplo, que, com relação ao setor saúde, na perspectiva de um município, o secretário de saúde já possui ao seu alcance um vasto rol de indicadores para serem agregados/desenvolvidos juntamente com o processo de orçamentação. Essa característica do setor saúde pode ser considerada como indicativa da possibilidade de uso do PB nesse setor.

Se considerarmos, no serviço público, a dispersão e a duplicação de ações, as práticas emergenciais que deveriam ser corriqueiras, os gastos que não indicam uma efetividade e o retorno à população, e que todas essas práticas são comuns, temos uma realidade de políticas públicas pouco impactantes para a melhoria das condições de vida da população. Muitas vezes, para dar cumprimento a comandos legais, os entes públicos realizam despesas com total descompromisso com a efetividade e o custo-benefício do gasto. Sendo assim, os orçamentos são relevantes instrumentos para alcançar esses objetivos.

Nesse contexto, Nóbrega (2011) destaca, ao analisar o PB:

O Performance Budget não pode ser encarado com um fim em si mesmo, mas como uma etapa de um amplo processo de reforma do setor público e que o sucesso dessa técnica depende não apenas de seu acurado aspecto técnico mas sim de uma ampla gama de fatores como o ambiente político, instituições e a situação fiscal do país. (P. 719). 
Já Robinson (2008), em seu seminário, apresenta-o como um procedimento ou mecanismo, ligando os fundos providos pelo setor público e os resultados alcançados, considerando, para tanto, a informação sobre o desempenho dos programas de governo e a utilização dessa informação pelos tomadores de decisão. O principal objetivo do PB é aprimorar a alocação e a eficiência dos recursos públicos. Assim, a informação sobre o desempenho dos gastos e os recursos alocados são funções centrais para esse tipo de orçamento.

É de suma importância indicar que não há, de fato, uma metodologia específica de análise sobre o que se entende por 'desempenho' para as políticas públicas de saúde e, ao mesmo tempo, para os instrumentos orçamentários.

\section{Conclusão}

O conceito de desempenho, nas suas mais variadas formas identificadas na análise desenvolvida, levou à organização de vários tipos de indicadores numa matriz que pudesse responder se as políticas públicas de saúde existentes em São Bernardo do Campo geraram, de fato, melhora das condições de vida da população.

Ao se avançar no estudo realizado, objetivando uma avaliação de como foram os processos de gestão da saúde pública no município de São Bernardo do Campo, identificou-se uma mudança, para melhor, a partir do início da gestão do segundo grupo gestor (2009-2012), que desencadeou uma real organização do sistema de saúde municipal.

Constatou-se que esse segundo grupo gestor assegurou o maior número de cumprimentos de metas pactuadas para as ações (estabelecidas no processo orçamentário), garantindo a expansão das equipes e da rede de serviços de saúde pública.

Quando, então, consolidadas as tabelas (1, 2, 3 e 4) de avaliação segundo as categorias do Orçamento por Desempenho - PB (Recursos, Outputs, Eficiência e Outcomes), incluindo 33 indicadores selecionados pelos processos de pactuação do SUS, no período de 2006 a 2012, permitiu-se a efetivação de uma avaliação de desempenho.

Para além de uma nova e simples reclassificação dos indicadores, o esforço se deu em conciliar as subfunções orçamentárias e, com certa dificuldade, os valores orçamentário-financeiros despendidos ao longo dos anos.

É fundamental ressaltar que os gestores públicos devem se preocupar, fundamentalmente, com a melhor elaboração dos instrumentos orçamentários para possibilitar o uso da ferramenta Orçamento por Desempenho. Como evidenciado, o segundo grupo gestor da saúde em São Bernardo do Campo aumentou a possibilidade do uso do PB em seu processo de orçamentação, porém, não o estruturou de forma completa (como proposto neste trabalho).

Assim, um novo desafio se apresenta quando se considera que os programas organizados nos PPAs, suas LDOs e LOAs tenham seus recursos realocados/reorganizados a partir dos desempenhos alcançados por cada ação proposta. Esse é o ponto fundamental de um novo método que envolve a responsabilização dos gestores sobre as políticas de saúde, que devem ser acompanhadas a partir de seus desempenhos. 


\section{Referências}

BONACIM, C. A. G.; ARAUJO, A. M. P. Avaliação de desempenho econômico-financeiro dos serviços de saúde. Ciência Saúde Coletiva, Rio de Janeiro, 16, Supl. 1, p. 1055-1068, 2011.

BRASIL. Ministério da Saúde. Gabinete do Ministro. Portaria ${ }^{\circ} 399$, de 22 de fevereiro de 2006. Divulga o Pacto pela Saúde 2006 - Consolidação do SUS e aprova as Diretrizes Operacionais do Referido Pacto. Diário Oficial [da] União, Brasília, DF, 22 fev. 2006. Disponível em: <http://portal2.saude.gov.br/saudelegis/LEG_

NORMA_PESQ_CONSULTA.CFM $>$. Acesso em: $22 \mathrm{fev}$. 2013.

. Presidência da República. Casa Civil. Decreto $\mathrm{n}^{\mathrm{o}} 7.508$, de 28 de junho de 2011. Regulamenta a Lei no 8.080, de 19 de setembro de 1990, para dispor sobre a organização do Sistema Único de Saúde - SUS, o planejamento da saúde, a assistência à saúde e a articulação interfederativa, e dá outras providências. Diário Oficial [da] União, Brasília, DF, 28 jun. 2011. Disponível em: <http://www.planalto.gov.br >. Acesso em: 15 jan. 2013.

Presidência da República. Casa Civil. Lei no 8.080, de 19 de setembro de 1990a. Dispõe sobre as condições para a promoção, proteção e recuperação da saúde, a organização e o funcionamento dos serviços correspondentes e dá outras providências. Diário Oficial [da] União, Brasília, DF, 19 set. 1990. Disponível em: <http://www.planalto.gov.br>. Acesso em: 10 nov. 2012.

Presidência da República. Casa Civil. Lei n ${ }^{\circ}$ 8.142, de 28 de dezembro de 1990b. Dispõe sobre a participação da comunidade na gestão do Sistema Único de Saúde (SUS) e sobre as transferências intergovernamentais de recursos financeiros na área da saúde e dá outras providências. Diário Oficial [da] União, Brasília, DF, 28 dez. 1990. Disponível em: <http://www.planalto.gov.br>. Acesso em: 10 nov. 2012.

Tribunal de Contas da União. Técnicas de Auditoria: Indicadores de Desempenho e Mapa de Produtos. Brasília, DF: TCU, 2000. Disponível em:
<http://portal3.tcu.gov.br/portal/page/portal/TCU/ comunidades/programas_governo/tecnicas_anop/ INDICADOR_DESEMPENHO_MAPA_PRODUTOS. pdf>. Acesso em: 20 jul. 2016.

CAMPOS, G. W. S. et al. Considerações sobre a Arte e a Ciência da Mudança: Revolução das Coisas e Reforma das Pessoas. In: CECÍLIO, L. C. O. (Org.). Inventando a Mudança na Saúde. São Paulo: HUCITEC, 1994. p. 29-87.

COSTA, J. M. B. S. et al. Desempenho de intervenções de saúde em países da América Latina: uma revisão sistemática. Saúde em Debate, Rio de Janeiro, v. 39, n. esp., dez. 2015.

MENDES, A. N.; SANTOS, S. B. S. Aperfeiçoamento em Administração Orçamentária e Financeira Governamental. São Paulo: Secretaria de Estado dos Negócios da Fazenda, Editora Fazesp, 2001.

MICLOS, P. V.; CALVO, M. C. M.; COLUSSI, C. F. Avaliação do desempenho da Atenção Básica nos municípios brasileiros com indicador sintético. Saúde em Debate, Rio de Janeiro, v. 39, n. 107, out./dez. 2015.

NOBREGA, M. Orçamento, eficiência e performance budget. In: SCAFF, F. F.; CONTI, J. M. (Org.). Orçamentos públicos e direito financeiro. São Paulo: Revista dos Tribunais, 2011.

ORGANIZATION FOR ECONOMIC CO-OPERATION AND DEVELOPMENT. Performance budgeting in OECD countries. Paris: OECD, 2007. Disponível em: <http://www.planejamento.gov.br/secretarias/ upload/Arquivos/seges/arquivos/ocde2011/oecd performance_countries.pdf $>$. Acesso em: 20 jul. 2016

PAIM, J. S.; TEIXEIRA, C. F. Política, planejamento e gestão em saúde: balanço do estado da arte. Revista Saúde Pública, São Paulo, v. 40, n. esp., p. 73-8, 2006.

REIS, A. A. C. Entre a intenção e o ato: uma análise da política de contratualização dos hospitais de ensino (2004-2010). 2011. 433 f. Tese. (Doutorado em Saúde 
Coletiva) - Universidade Federal de São Paulo, São Paulo, 2011.

ROBINSON, M. Orçamento por Desempenho $(O D)$ : modelos de orçamento por resultado e fatores chaves. São Paulo, 2008. Disponível em: $<$ http://www.fazenda.sp.gov.br/seminario_ orcamento\%5C0112\%5Cpainel3_0112_Marc_Robinson. pdf >. Acesso em: 20 fev. 2013.
SANTOS JR., D. P. Gestão participativa e a reestruturação da rede municipal de saúde em São Bernardo do Campo, SP, no período de 2009 a 2011. 2011. 139f. Dissertação (Mestrado em Saúde Pública) Universidade de São Paulo, São Paulo, 2011.

Recebido para publicação em abril de 2016

Versão final em julho de 2016

Conflito de interesses: inexistente

Suporte financeiro: não houve 